\title{
An Unwonted, Pedagogic Case Report on Effort Thrombosis of Right Brachial Vein
}

\author{
Sayani Banerjee \\ Department of Emergency Medicine, Calcutta Medical Research Institute, Kolkata, India \\ Email: sayani.dr@gmail.com
}

How to cite this paper: Banerjee, S. (2022) An Unwonted, Pedagogic Case Report on Effort Thrombosis of Right Brachial Vein. Open Journal of Emergency Medicine, 10, 8-18.

https://doi.org/10.4236/ojem.2022.101002

Received: December 27, 2021

Accepted: February 14, 2022

Published: February 17, 2022

Copyright (c) 2022 by author(s) and Scientific Research Publishing Inc. This work is licensed under the Creative Commons Attribution International License (CC BY 4.0).

http://creativecommons.org/licenses/by/4.0/ (c) (i) Open Access

\begin{abstract}
Deep vein thrombosis (DVT) is one or more blood clots formed inside the deep vein in the body resulting in complete or partial blockage of blood flow through the affected vein. Upper Extremity DVT (UEDVT) accounts for 5\% $10 \%$ of all cases of DVTs. Previously it was thought to be a rare disorder. However in recent years with the advent of various indwelling intravenous devices, hypercoagulable state like COVID-19, secondary UEDVT did not remain infrequent presentation anymore. Though primary UEDVT, also known as Effort Thrombosis, that takes place without any underlying obvious pathology is a rare form till now. We presented a case of 46 years female who presented to the emergency with complaints of progressively increasing pain (7 days) and swelling ( 3 days) of her right forearm since last 7 days following strenuous and heavy work by her dominant/ right hand during the period of festival. She initially ignored her condition because of the Durga Puja festival. As her symptoms deteriorated she eventually had to attend the emergency department. Eventually an urgent ultrasonography color Doppler was done on emergency basis which diagnosed underlying DVT of her right brachial vein. She was managed with low molecular weight heparin, urgent fasciotomy owning to her impending compartment syndrome. Other tests ruled out any secondary underlying pathology. She improved and discharged without any complications on oral anticoagulant. Exigent events in her history were trauma followed by fall on her right elbow 2 months back and then presenting signs \& symptoms commencing at the same region following strenuous, tedious activities over 7 days and consequential effort thrombosis of her right brachial vein. All of that compelled us to ruminate on rare differentials of her presentation and eventually come to this rare diagnosis. The author hence brought this pedagogic case to the readers, especially emergency \& primary care physicians and emphasised the importance of being intuitive about rare but deadly differentials which come from proficiency \& experience in the field of medicine.
\end{abstract}




\section{Keywords}

Component, Formatting, Style, Styling, Insert

\section{Introduction}

Deep vein thrombosis (DVT) is one or more blood clots formed inside the deep vein in the body resulting in complete or partial blockage of blood flow through the affected vein [1]. DVT most commonly affects lower limbs \& thighs, pelvis. But upper extremity DVT (UEDVT) gains more importance recently owning to its high mortality \& morbidity secondary to its life threatening complication pulmonary embolism (PE) which is otherwise preventable if recognized and intervened early. UEDVT, consists of $5 \%-10 \%$ of all cases of DVT. PE, the most dreaded complication of any DVT, rates up to one third of the patients with UEDVT [2].

UEDVT are two types, primary and secondary. Primary UEDVT is a rare disorder (2 per 100,000 persons per year) [3] that refers to effort thrombosis, also called Paget-Schroetter Syndrome or idiopathic UEDVT. Patients with Paget-Schroetter Syndrome develop spontaneous UEDVT, usually in their dominant hand following repeated strenuous activity, like heavy weight lifting, wrestling, rowing etc. which causes micro trauma to the vessel intima resulting in activation of coagulation cascade and thrombosis [4]. Mechanical compression to the vein by adjacent muscle, tendon, and bone significantly increases the possibility of thrombosis in thoracic outlet obstruction, cervical ribs, long transverse processes of the cervical spine, musculofascial bands, and clavicle or first rib anomalies, athletes and body builders etc. [5] [6]. Patients with idiopathic UEDVT usually do not have any known trigger or obvious underlying disease. Secondary UEDVT accounts for most of the cases of UEDVT and patients with indwelling central venous catheter, cancer, and pacemaker are commonly prone to secondary UEDVT.

Prevalence of hypercoagulable states in secondary UEDVT is not known and previous observational studies have shown varying results so far. Cost effectiveness of screening UEDVT patients for coagulation disorders is also controversial and it is only proven to be fruitful in patients with idiopathic UEDVT, family history of DVT, recurrent unexplained pregnancy loss or prior DVT [7].

Most common veins involved in UEDVT are proximal subclavian, axillary veins. Though rare involvement of brachial and other distal veins has also been reported [8].

Clinical presentation of UEDVT includes pain, swelling, redness, and heaviness with or without numbness, paresthesia. On examination edema, erythema, engorgement of collateral veins, cyanosis may be present. Approximately $33 \%$ $60 \%$ patients may be asymptomatic on presentation [9] [10].

In case of suspected UEDVT compression ultrasonography (US) the preferred 
diagnostic modality is with a sensitivity of $97 \%$ and specificity of $96 \%$ [11] [12]. In cases of proximal UEDVT where compression US is not possible because of incompressibility attributed to anatomical location of the veins, subclavian \& brachiocephalic, Color Doppler US is used instead. Venography, when remains the gold standard to diagnose UEDVT, its use remains limited owing to its higher cost, availability and chances of radiation exposure [13].

In 2008 Constans et al. described clinical scoring system to determine probability of UEDVT in patients and in 2016 van Es et al. added d-dimer and US to create a diagnostic algorithm [14] [15] (Figure 1).

Treatment is targeted to dissolving the venous clot, by low molecular weight heparin (LMWH) or unfractionated heparin (UF) where LMWH is contraindicated. Fonda-perinox can be used instead in cases where LMWH/ UF is contraindicated, like heparin induced thrombocytopenia. Direct oral anticoagulants (DOAC) Rivaroxaban and Apixaban have been approved to treat LEDVT and can be used to treat UEDVT as well. Vitamin K antagonist (VKA) has been used as maintenance therapy with a target INR 2.0 - 3.0. Recently DOACs were employed in maintenance therapy of DVT on account of $40 \%$ reduced chance of major hemorrhage in comparison to VKA.

The initial treatment phase is 21 days for rivaroxaban and 7 days for apixaban, compared with 5 days for LMWH, UHF, and FDX. Maintenance therapy usually commenced for 3 - 6 months and can be prolonged depending upon underlying clinical condition [16].

\section{Case Presentation}

We present a case of 46 years female who presented to the emergency with complaints of progressively increasing pain (7 days) and swelling (3 days) of her right forearm since last. She ignored her pain and discomfort to right hand when it has started as she was busy with household work for last 2 weeks in festive season during Durga Puja. She specifically mentioned heavy weight lifting and exertion during this period of time. She is right hand dominant. Apart from her lumbar spine surgery 2 years ago, she did not mention any comorbidity. She was not on any regular medications during her presentation. She denied any chest pain, dyspnea, cough, hemoptysis, fever etc. She had no history of coagulation disorder/ thrombophilia/thromboembolism in her family or in her. At first interaction with her in ER she denied any trauma to her right upper limb. Though on day 4 of her admission during follow up visit to her she mentioned a fall followed by trauma to her right forearm 2 months ago, which she had never been evaluated for as her symptoms (only pain) resolved.

On examination, she was conscious, alert, oriented without any obvious neurodeficit. Blood pressure (BP) 130/90 $\mathrm{mmHg}$, heart rate (HR) 88/minute, oxygen saturation in room air $98 \%$, respiratory rate $16 /$ minute, capillary glucose 167 $\mathrm{mg} / \mathrm{dl}$, temperature 97.6 degree $\mathrm{F}$.

The examination of HEENT, lungs, heart, abdomen and lower extremities was normal. 


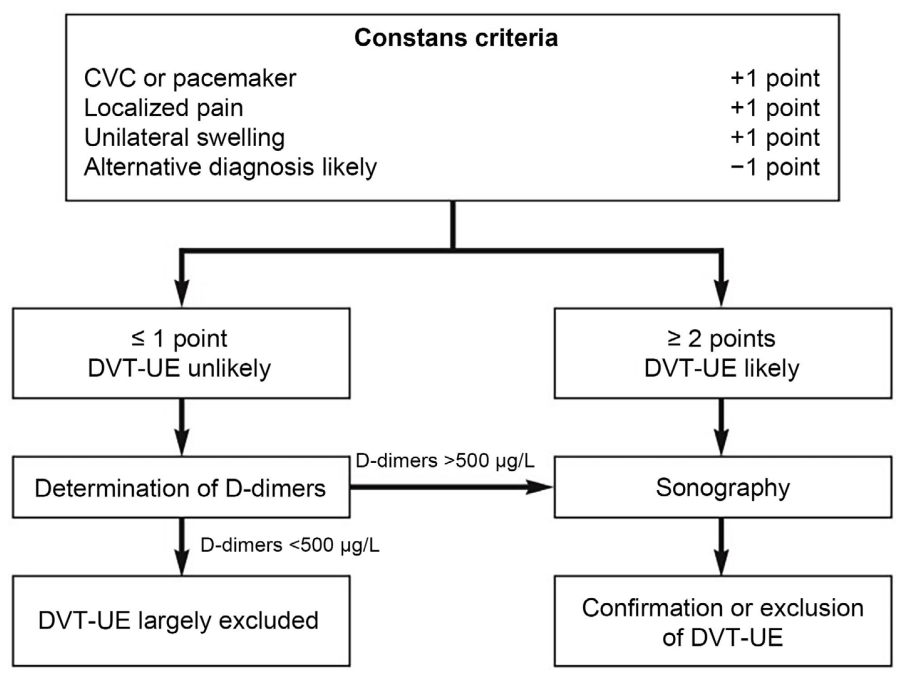

Figure 1. Diagnostic algorithm based on the Constans criteria [14] [15].

A focus examination of right upper extremity showed painful tense swelled up right forearm with intact peripheral pulses and sensation. Local rise of temperature, redness were noticed. There was no evidence of arterial insufficiency or neurological deficit. There was no axillary or supraclavicular lymphadenopathy. An urgent Doppler Ultrasonography of right upper limb done which confirmed deep vein thrombosis (DVT) involving short segment of right brachial vein in the elbow region. Diffuse subcutaneous edema noted involving entire right forearm. No evidence of DVT in right subclavian, axillary, radial or ulnar vein. Arterial Doppler of right upper limb was within normal limits.

Laboratory investigations revealed:

Creatinine $0.56 \mathrm{mg} / \mathrm{dl}$, Potassium 4.46, sodium $136.5 \mathrm{mEq} / \mathrm{L}$, Urea $12 \mathrm{mg} / \mathrm{dl}$, CRP 0.63, Viral Serology negative, HB $8.6 \mathrm{gm} / \mathrm{dl}$, total Leukocyte count 7500/cumm (N63 L30 B0 M3), ESR 20, RBC 3.73 RDW 16 MCH 23 MCHC 30.6 MCV 75 PCV 28.

Peripheral smear suggestive of (s/o) Microcytic hypochromic red blood cells, white blood cells (Total \& differential counts) adequate number with normal morphology, platelets are adequate (Platelets 2,40,000/cumm).

Her Chest X-ray (AP view) showed no obvious abnormality. Her right elbow \& forearm X-ray revealed no radiological abnormality. Patient was immediately started on anticoagulant low molecular weight heparin, intravenous antibiotic, analgesic and other supportive treatment followed by fasciotomy of right upper limb in view of impending compartment syndrome. Her local wound swab for gram stain and culture-sensitivity came to be sterile and ruled out any underlying associated cellulitis. Patient improved and discharged on oral anticoagulant.

\section{Case Discussion}

We present a rare case of effort thrombosis of right brachial vein in a 46 years old woman. Effort thrombosis usually presents with sudden, severe pain and 
swelling of upper extremity due to thrombosis of veins of upper extremity secondary to strenuous and repetitive activities of upper limb. It involves axillary and subclavian veins mostly but rarely brachial and other peripheral veins are also involved. Effort thrombosis accounts for $30 \%-40 \%$ of spontaneous axillary \& subclavian vein thrombosis alias Paget-Schroetter Syndrome (PSS) or venous thoracic outlet syndrome (VTOS), and for $10 \%-20 \%$ of all upper extremity deep venous thrombosis (UEDVT) [17].

\subsection{Pathogenesis of Effort Thrombosis}

Vigorous, sustained upper extremity movements, retroversion, hyper abduction, extension, like some sports, for example: wrestling, swimming, gymnastics, bowling etc. results in peri-venous micro trauma activating intrinsic coagulation cascade and eventually thrombosis. Anatomical abnormalities involving the thoracic outlet (cervical rib, congenital bands, hypertrophy of scalenus tendons and abnormal insertion of the costoclavicular ligament) play a pivotal role in the pathogenesis of effort thrombosis [18]. Inflammation leading to perivenular fibrosis, adhesions perpetuates the obstruction further. The role of inherited and acquired thrombophilic disorders remains unresolved and needs further investigation. It is not cost effective to the patient who does not show past thrombophilic tendencies or significant family history. So clinicians in India often omit these tests.

Our middle aged, apparently well, right hand dominant female, housewife presented to us with history of repetitive strenuous work at her home during the past one week. To avoid causing trouble to her family members and relatives who came to her place for Durga Puja festival, she ignored her symptoms until it became obvious, unendurable. She denied any history of previous bleeding/ clotting tendencies, coagulation disorder, past history of any blood/ blood product transfusion or any family history of same/ known coagulopathy. Initially on her presentation she did not mention any trauma. Though on day 3 of her hospitalization she mentioned a fall two months back followed by trauma on her right elbow. She never consulted any physician nor was she investigated for that as her pain subsided on its own. There was no proper way to be sure but the possibility of micro trauma and brachial vein intimal weakening resulting from trauma to her right hand two month back and spontaneous thrombosis of her right brachial vein triggered by the recent vigorous activity by her dominant/ right hand.

Youssef, M. Y. Z., et al. (2006) reported 3 cases of effort thrombosis following a session of intense weight lifting. In the 1st case, the lesion was bilateral and complicated by superior vena cava obstruction. In the 2 nd and in the 3 rd case, right and left subclavian vein thrombosis, respectively. All cases were managed with oral anticoagulation therapy after consultation with vascular surgery team [19]. Ijaopo, R. et al. (2016) reported a 37-year-old Caucasian man, an active judo tutor who initially presented to his primary care physician complaining of redness and swelling of his right arm and elbow of 1 week's duration. He had 
been prescribed some antibiotics by his general practitioner, but as his symptoms worsened he was referred to the hospital acute care unit where working diagnosis was possible olecranon bursitis and right arm cellulitis to rule out DVT was made. Venous Doppler ultrasonography revealed a right axillary DVT with extension into the cephalic and brachial veins. Vascular team was involved and he subsequently undergone catheter directed thrombolysis followed by anticoagulation. In this case report authors emphasised the importance of awareness of rare causes of PSS and consideration of risk factors like age, hobby, occupation etc. of PSS among general \& primary care physicians to avoid missing a diagnosis, its complications and need for any invasive interventions [20]. Jacob, Vinitha et al. described a 40-year-old right-hand-dominant male bodybuilder presented for evaluation of right upper extremity swelling noticed for 3 days. His medical and social history was significant for regular injection use of testosterone as well as an active 30 pack-year smoking history. US image shows a thrombus in the right subclavian vein. A heparin drip was started, and the vascular surgery service was consulted from the emergency department. This patient underwent successful catheter directed thrombolysis. Even with anticoagulation and thrombolysis, re-thrombosis can occur in as many as a third of patients. Due to the high risk of re-thrombosis, in this case report author emphasised on definitive treatment of Paget-Schroetter disease, decompression surgery. As the treatment of UEDVT is time sensitive, it is crucial for the emergency physicians to be familial of the management of UEDVT, which is distinct in compared to lower limb DVT and to involve the appropriate specialists in an expeditious manner [21].

In literature we found most cases of effort thrombosis described in athletes mimicking common musculoskeletal disorders. Emergency and primary care physicians should keep in mind that timely and early interventions, investigations and initiation of treatment are the utmost important factors to determine the prognosis and functional restoration, preventing dreaded complications like pulmonary embolism. Most common veins involved were axillary and subclavian veins. Isolated brachial vein involvement is extremely rare and hardly reported in previous literatures.

\subsection{Clinical Presentation \& Diagnosis}

Common presentations of effort thrombosis/ UEDVT are same as any other DVT: heaviness, discomfort, pain, paresthesia and swelling of the affected arm, all of which were described by our patient on presentation. Physical Examination may reveal: edema, redness, cyanosis, visible collateral veins and even fever/ local rise of temperature of the affected arm [9]. Apart from edema, erythema, redness of right forearm, our patient did in fact had local rise of temperature which could have been a misguiding sign towards the differential of cellulitis. Fortunately, we developed a practice of ruling out DVT in cases of all cellulitis owning to high incidences of associated DVT in our settings. It was a correct 
judgment call to order an US color Doppler of the affected limb, which revealed correspondingly UEDVT of right brachial vein. Subsequent blood tests, gram stain \& C/S of local wound swab averted cellulitis as a differential diagnosis. When venography remains the gold standard diagnostic modality, it is largely being replaced by compression \& color Doppler ultrasonography, having sensitivity of $97 \%$ \& specificity of $96 \%$ [9] [13]. Contrast enhanced (CE) computed tomography (CT) or magnetic resonance imaging (MRI) not only can confirm UEDVT but also diagnose the underlying pathologies like cancer, anatomical abnormalities etc.

\subsection{Management}

Treatment of primary or effort thrombosis is time sensitive and distinct from lower limb DVT. Emergency \& primary care physicians must be coherent with the management of UEDVT to prevent rare but dreaded complications like PE. It is also crucial to involve the appropriate specialists like vascular surgeon in an expeditious manner. Anticoagulation is the mainstay of treatment, to start with LMWH or Fondaparinux for 5 days and then to continue with oral anticoagulants [vitamin $\mathrm{K}$ antagonist, direct thrombin inhibitors] for at least 3 months. In patients with renal failure or undergoing invasive treatment modalities unfractionated heparin is used instead [22]. Compression stocking are useful after the acute phase is over. Superior vena cava filters are hardly recommended attributed to its grave complications (cardiac tamponed, aortic perforation). Catheter directed thrombectomy or thrombolysis (CDT) are reserved for refractory cases, cases with more proximal UEDVT, presenting with severe symptoms. Paget-Schroetter Syndrome needs multidisciplinary approach in management consisting of anticoagulation therapy, CDT, or pharmaco-mechanical thrombolysis and subsequent surgical correction of VTOS, physiotherapy etc. Even with anticoagulation and thrombolysis, re-thrombosis can occur in as many as one third of patients. High risk of re-thrombosis necessitates definitive treatment, decompression surgery. Patients who were aggressively treated with this approach (thrombolysis and decompression surgery) reported quicker resolution of symptoms and return to work than those treated with anticoagulation alone [18].

Our patient was started on low molecular weight heparin, antibiotic, analgesic and other supportive medications from emergency department. She was admitted under plastic surgeon and fasciotomy was done in view of impending compartment syndrome secondary to soft tissue edema, limb swelling. Local tissue swab gram stain \& culture sensitivity came negative which ruled out any associated cellulitis. Her HB concentration was slightly lower than normal. Apart from that all other routine blood reports, coagulation profile came to be normal. Patient improved and discharged on day 7 on oral anticoagulant for next 3 months. On follow up she showed no signs of complications/ recurrence (Figure 2). 


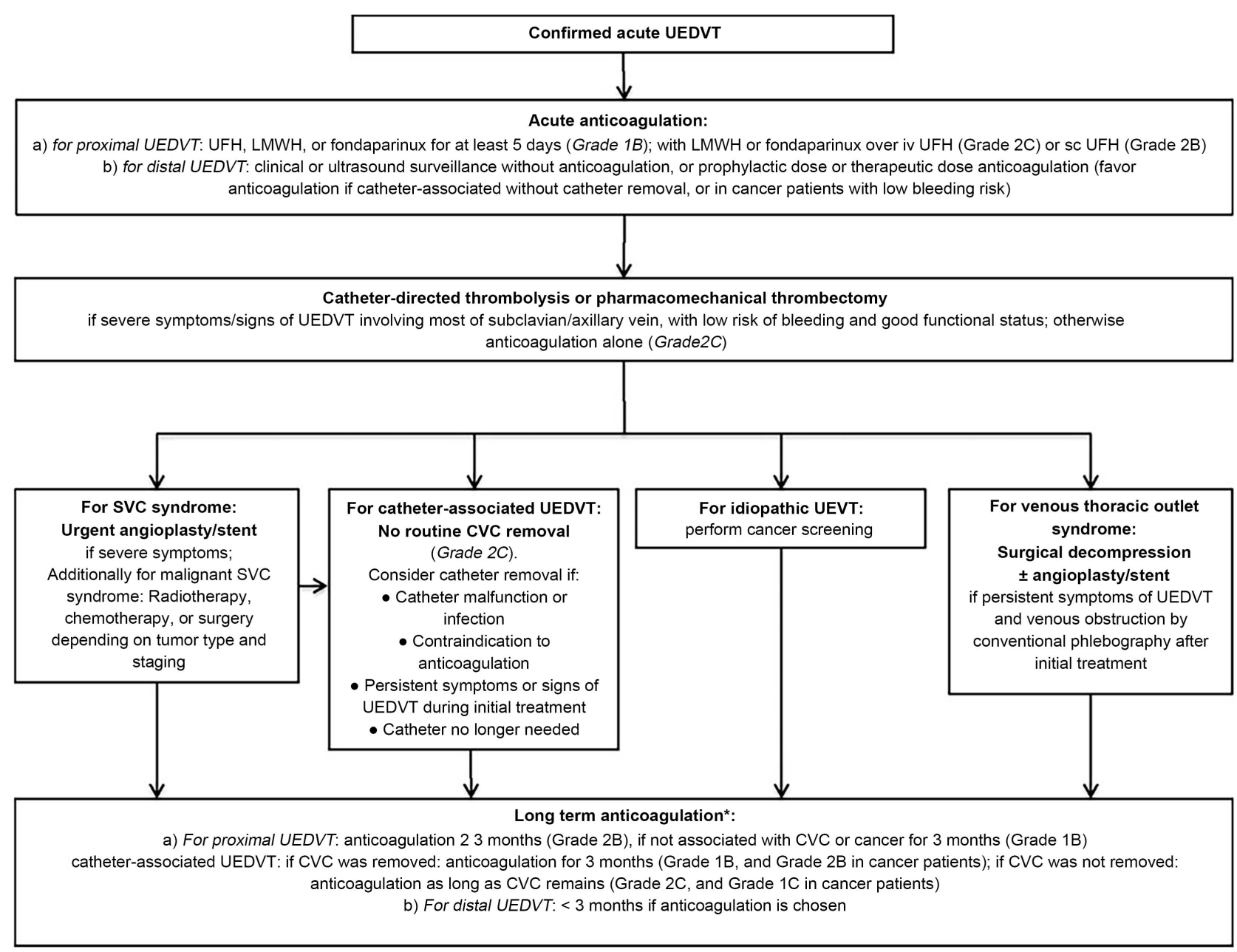

Figure 2. Suggested algorithm for management of UEDVT proposed by American College of Chest Physician in 2012 Consensus [17]. Levels of evidence graded as follows: Grade 1B indicates strong recommendation with moderate-quality evidence; grade 2B, weak recommendation with moderate-quality evidence; and grade $2 \mathrm{C}$, low- or very-low-quality evidence. CVC: central venous catheter; LMWH: low-molecular-weight heparin; SVC: superior vena cava; and UFH: unfractionated heparin. ${ }^{\star}$ Includes vitamin $\mathrm{K}$ antagonist, LMWH, dabigatran, or rivaroxaban.

\subsection{Complications of UEDVT/ Effort Thrombosis}

- Up to one third of the patients with UEDVT may develop pulmonary embolism (PE). Other complications like post thrombotic syndrome, recurrent thromboembolism may also occur [2].

- Lymphedema, chronic venous insufficiency lead to compartment syndrome, like in our patient, which prompted urgent surgical intervention, fasciotomy.

- Neuropathy, brachial plexopathy.

- Septic thrombophlebitis.

- Superior Vena Cava (SVC) syndrome etc.

\section{Conclusion}

We concluded that our patient had UEDVT \& effort thrombosis of right brachial vein which was acutely precipitated by constant strenuous work at home in- 
cluding heavy weight lifting in the last week. Ignoring her symptoms complicated her presentation to us with impending compartment syndrome mimicking a cellulitis. The UEDVT is rare and it could easily be missed unless high suspicion by emergency \& primary care physicians, especially as it can mimic many other common conditions and acute presentations like cellulitis. Previously it was thought to be a rare disorder. However in recent years with the advent of various indwelling intravenous devices, hypercoagulable state like COVID-19, secondary UEDVT did not remain infrequent presentation anymore. Though primary UEDVT, also known as Effort Thrombosis, that takes place without any underlying obvious pathology is a rare form till now. Exigent events in her history were trauma followed by fall on her right elbow 2 months back and then presenting signs \& symptoms commencing at same region following strenuous, tedious activities over 7 days and consequential effort thrombosis of her right brachial vein. All of that compelled us to ruminate on rare differentials of her presentation and eventually come to this rare diagnosis. The author hence brought this pedagogic case to the readers, especially emergency \& primary care physicians and emphasised the importance of being intuitive about rare but deadly differentials which come from proficiency \& experience in the field of medicine.

\section{Acknowledgements}

The anonymous patients who teach us every day, thanks.

\section{Conflicts of Interest}

The author declares no conflicts of interest regarding the publication of this paper.

\section{References}

[1] Piazza, G. and Goldhaber, S.Z. (2006) Acute Pulmonary Embolism: Part I: Epidemiology and Diagnosis. Circulation, 114, e28-e32. https://doi.org/10.1161/CIRCULATIONAHA.106.620872

[2] Prandoni, P., Polistena, P., Bernardi, E., Cogo, A., Casara, D., Verlato, F., et al. (1997) Upper-Extremity Deep Vein Thrombosis: Risk Factors, Diagnosis and Complications. JAMA Internal Medicine, 157, 57-62. https://doi.org/10.1001/archinte.1997.00440220061008

[3] Lindblad, B., Tengborn, L. and Bergqvist, D. (1988) Deep Vein Thrombosis of the Axillary-Subclavian Veins: Epidemiologic Data, Effects of Different Types of Treatment and Late Sequelae. European Journal of Vascular Surgery, 2, 161-165. https://doi.org/10.1016/S0950-821X(88)80069-0

[4] Zell, L., Kindermann, W., Marschall, F., Scheffler, P., Gross, J. and Buchter, A. (2001) Paget-Schroetter Syndrome in Sports Activities: Case Study and Literature Review. Angiology, 52, 337-342. https://doi.org/10.1177/000331970105200507

[5] Thompson, R.W., Schneider, P.A., Nelken, N.A., Skioldebrand, C.G. and Stoney, R.J. (1992) Circumferential Venolysis and Paraclavicular Thoracic Outlet Decompression for "Effort Thrombosis" of the Subclavian Vein. Journal of Vascular Sur- 
gery, 16, 723-732. https://doi.org/10.1016/0741-5214(92)90227-Y

[6] Parziale, J.R., Akelman, E., Weiss, A.P. and Green, A. (2000) Thoracic Outlet Syndrome. American Journal of Orthopedics, 29, 353-360.

[7] Ruggeri, M., Castaman, G., Tosetto, A. and Rodeghiero, F. (1997) Low Prevalence of Thrombophilic Coagulation Defects in Patients with Deep Vein Thrombosis of the Upper limbs. Blood Coagulation \& Fibrinolysis, 8, 191-194. https://doi.org/10.1097/00001721-199704000-00006

[8] Hingorani, A., Ascher, E., Marks, N., Schutzer, R.W., Mutyala, M., Yorkovich, W., et al. (2006) Morbidity and Mortality Associated with Brachial Vein Thrombosis. Annals of Vascular Surgery, 20, 297-300. https://doi.org/10.1007/s10016-006-9040-0

[9] Kucher, N. (2011) Clinical Practice Deep-Vein Thrombosis of the Upper Extremities. New England Journal of Medicine, 364, 861-869. https://doi.org/10.1056/NEJMcp1008740

[10] Sajid, M.S., Ahmed, N., Desai, M., Baker, D. and Hamilton, G. (2007) Upper Limb Deep Vein Thrombosis: A Literature Review to Streamline the Protocol for Management. Acta Haematologica, 118, 10-18. https://doi.org/10.1159/000101700

[11] Baarslag, H.-J., van Beek, E.J.R., Koopman, M.M.W. and Reekers, J.A. (2002) Prospective Study of Color Duplex Ultrasonography Compared with Contrast Venography in Patients Suspected of Having Deep Venous Thrombosis of the Upper Extremities. Annals of Internal Medicine, 136, 865.

https://doi.org/10.7326/0003-4819-136-12-200206180-00007

[12] Di Nisio, M., Van Sluis, G.L., Bossuyt, P.M.M., Büller, H.R., Porreca, E. and Rutjes, A.W.S. (2010) Accuracy of Diagnostic Tests for Clinically Suspected Upper Extremity Deep Vein Thrombosis: A Systematic Review. Journal of Thrombosis and Haemostasis, 8, 684-692. https://doi.org/10.1111/j.1538-7836.2010.03771.x

[13] Di Nisio, M., van Sluis, G.L., Bossuyt, P.M., Buller, H.R., Porreca, E. and Rutjes, A.W. (2010) Accuracy of Diagnostic Tests for Clinically Suspected Upper Extremity Deep Vein Thrombosis: A Systematic Review. Journal of Thrombosis and Haemostasis, 8, 684-692. https://doi.org/10.1111/j.1538-7836.2010.03771.x

[14] van Es, N., Bleker, S.M., Di Nisio, M., Kleinjan, A., Beyer-Westendorf, J., Camporese, G., et al. (2016) A Clinical Decision Rule and D-Dimer Testing to Rule out Upper Extremity Deep Vein Thrombosis in High-Risk Patients. Thrombosis Research, 148, 59-62. https://doi.org/10.1016/j.thromres.2016.10.019

[15] Constans, J., Salmi, L.R., Sevestre-Pietri, M.A., Perusat, S., Nguon, M., Degeilh, M. et al. (2008) A Clinical Prediction Score for Upper Extremity Deep Venous Thrombosis. Thrombosis and Haemostasis, 99, 202-207. https://doi.org/10.1160/TH07-08-0485

[16] AWMF (Arbeitsgemeinschaft der Wissenschaftlichen Medizinischen Fachgesellschaften e.V.) (2015) S2k-Diagnostik und Therapie der Venenthrombose und der Lungenembolie.

[17] Bernardi, E., Pesavento, R. and Prandoni, P. (2006) Upper Extremity Deep Venous Thrombosis. Seminars in Thrombosis and Hemostasis, 32, 729-736. https://doi.org/10.1055/s-2006-951458

[18] Urschel Jr., H.C. and Patel, A.N. (2008) Surgery Remains the Most Effective Treatment for Paget-Schroetter Syndrome: 50 Years' Experience. The Annals of Thoracic Surgery, 86, 254-260. https://doi.org/10.1016/j.athoracsur.2008.03.021

[19] Youssef, M.Y., Al Taweel, T., Asfar, S. and Abdella, N. (2006) Effort-Induced Venous Thrombosis of the Upper Limbs. Medical Principles and Practice, 15, 299-302. 
https://doi.org/10.1159/000092994

[20] Ijaopo, R., Oguntolu, V., DCosta, D., Garnham, A. and Hobbs, S. (2016) A Case of Paget-Schroetter Syndrome (PSS) in a Young Judo Tutor: A Case Report. Journal of Medical Case Reports, 10, Article No. 63.

https://doi.org/10.1186/s13256-016-0848-0

[21] Jacob, V., Daley, J. and Coughlin, R.F. (2021) The Diagnosis and Management of Paget-Schroetter Syndrome (Primary Effort Thrombosis) in the Emergency Department. Medicine Case Reports and Study Protocols, 2, Article No. e0106. https://doi.org/10.1097/MD9.0000000000000106

[22] Kearon, C., Akl, E.A., Comerota, A.J., Prandoni, P., Bounameaux, H., Goldhaber, S.Z., Nelson, M.E., Wells, P.S., Gould, M.K., Dentali, F., Crowther, M. and Kahn, S.R. (2012) Antithrombotic Therapy for VTE Disease: Antithrombotic Therapy and Prevention of Thrombosis, 9th Edition: American College of Chest Physicians Evidence-Based Clinical Practice Guidelines. Chest, 141, e419S-e494S.

https://doi.org/10.1378/chest.11-2301 